\title{
On the Effect of Step-Doped Quantum Barriers in InGaN/GaN Light Emitting Diodes
}

\author{
Zi-Hui Zhang, Swee Tiam Tan, Zhengang Ju, Wei Liu, Yun Ji, Zabu Kyaw, Yilmaz Dikme, \\ Xiao Wei Sun, Senior Member, IEEE, and Hilmi Volkan Demir
}

\begin{abstract}
InGaN/GaN light-emitting diodes (LEDs) make an important class of optoelectronic devices, increasingly used in lighting and displays. Conventional InGaN/GaN LEDs of c-orientation exhibit strong internal polarization fields and suffer from significantly reduced radiative recombination rates. A reduced polarization within the device can improve the optical matrix element, thereby enhancing the optical output power and efficiency. Here, we have demonstrated computationally that the step-doping in the quantum barriers is effective in reducing the polarization-induced fields and lowering the energy barrier for hole transport. Also, we have proven experimentally that such InGaN/GaN LEDs with $\mathrm{Si}$ step-doped quantum barriers indeed outperform LEDs with wholly Si-doped barriers and those without doped barriers in terms of output power and external quantum efficiency. The consistency of our numerical simulation and experimental results indicate the effects of $\mathrm{Si}$ step-doping in suppressing quantum-confined stark effect and enhancing the hole injection, and is promising in improving the InGaN/GaN LED performance.
\end{abstract}

Index Terms-InGaN, GaN, light-emitting diode (LED), quantum-confined Stark effect (QCSE), Si-doping.

\section{INTRODUCTION}

$\mathbf{T}$ ARGETTING for general lighting, a significant progress in $\mathrm{InGaN} / \mathrm{GaN}$ light-emitting diodes (LEDs) has been made since the first InGaN/GaN-based LED was demonstrated [1]. Typically, a high external quantum efficiency (EQE) is

Manuscript received May 24, 2012; revised May 30, 2012; accepted May 31, 2012. Date of publication July 18, 2012; date of current version March 15, 2013. This work is supported by the Singapore National Research Foundation under Grant NRF-RF-2009-09 and Grant NRF-CRP-6-2010-2, and by the Singapore Agency for Science, Technology and Research (A*STAR) SERC under Grant 1121202009.

Z.-H. Zhang, S. T. Tan, Z. G. Ju, W. Liu, Y. Ji, and Z. Kyaw are with LUMINOUS! Center of Excellence for Semiconductor Lighting and Displays, School of Electrical and Electronic Engineering, Nanyang Technological University, 639798 Singapore (e-mail: zhan0340@ntu.edu.sg; sweetiam@ntu.edu.sg; zgju@ntu.edu.sg; liu_wei@ntu.edu.sg jiyu0004@ntu.edu.sg; zinm0013@ntu. edu.sg).

Y. Dikme is with AIXaTech GmbH, 52074 Aachen, Germany (e-mail: y.dikme@aixatech.com).

X. W. Sun is with LUMINOUS! Center of Excellence for Semiconductor Lighting and Displays, School of Electrical and Electronic Engineering, Nanyang Technological University, 639798 Singapore (e-mail: exwsun@ntu.edu.sg).

H. V. Demir is with LUMINOUS! Center of Excellence for Semiconducto Lighting and Displays, School of Electrical and Electronic Engineering, School of Physical and Mathematical Sciences, Nanyang Technological University, 639798 Singapore, and also with the Department of Electrical and Electronics Engineering, Department of Physics, UNAM - Institute of Materials Science and Nanotechnology, Bilkent University, Bilkent, Ankara 06800 Turkey (e-mail: volkan@stanfordalumni.org).

Color versions of one or more of the figures are available online at http:// ieeexplore.ieee.org.

Digital Object Identifier 10.1109/JDT.2012.2204858 observed from InGaN/GaN LEDs under low current injection levels. However, the efficiency is substantially reduced under elevated current injection, and yet high current is required in most of the lighting applications [2]. This effect is well recognized as the efficiency droop. Various models have thus far been proposed to explain the droop, such as junction heating [3], electron overflow [4], [5], reduced effective radiative recombination rate due to the elevated plasma temperature caused by carrier-carrier and carrier-photon collisions [5], current crowding [6] and Auger recombination [7].

To date, several methods have been suggested to improve the efficiency and enhance the optical output power. For example, Lee et al. enhanced the efficiency by grading InN fraction in InGaN quantum well structures [8]. Wang et al. improved the efficiency by incorporating quantum wells with graded thickness [9]. Zhao et al. employed thin AlInN barriers to suppress the thermionic carrier escape rate [10]. Additionally, electron blocking layers (EBL) that improve the emission of InGaN/GaN LEDs, including stepwise-stage EBL [11], p-type AlGaN/GaN superlattice with a graded AlN composition [12], AlGaN EBL with graded AlN fraction [13], and even AlInN EBL [14] has also been studied. Recently, it has been shown that three-dimensional hole gas [15] is effective in increasing the hole concentration, thus enhancing the optical power.

Besides improving the carrier injection efficiency, it is necessary to increase the electron-hole wave function overlap $\left(\Gamma_{\mathrm{e}-\mathrm{hh}}\right)$. For that, staggered InGaN quantum wells have been proposed and investigated [16]-[20]. The spatial separation of the electron-hole wave functions can be reduced also by employing either the ternary InGaN substrate [21] or the electro-plated Ni metal substrate [22]. Recently, c-plane III-Nitride quantum wells with embedded "delta" novel materials have proved to be effective in enhancing the electron-hole wave function overlap, therefore increasing the radiative recombination rates [23]-[26]. The strain induced spatial separation of electron-hole wave functions can further be completely eliminated in the non-polar quantum wells and increased radiative recombination rates can thus be obtained [27], [28]. It has also been shown that the material quality and the device performance can be substantially improved by introducing Si doping in quantum barriers [29]-[31]. However, Si-doped barriers or even Si-delta-doped barriers usually have a setback from holes blocking [32], [33], which leads to a high local hole accumulation. Previously, Zhu et al. proposed the selective $\mathrm{Si}$ doped barriers to symmetrize the hole distribution and improve the LED performance [34]. Nevertheless, selective Si doping could not effectively suppress the polarizations in those quantum wells due to the undoped quantum barriers. On the other hand, it has been reported that the free electrons (released 


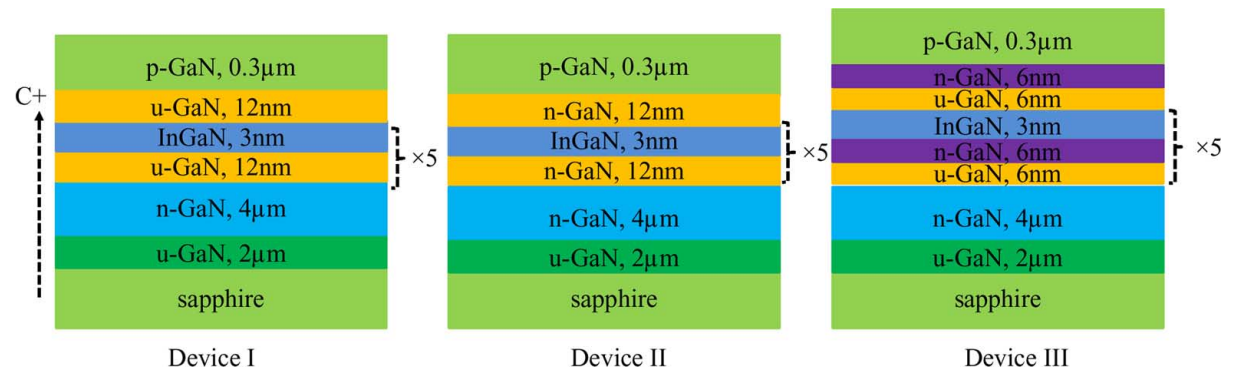

Fig. 1. Schematic diagrams of the studied LED structures.
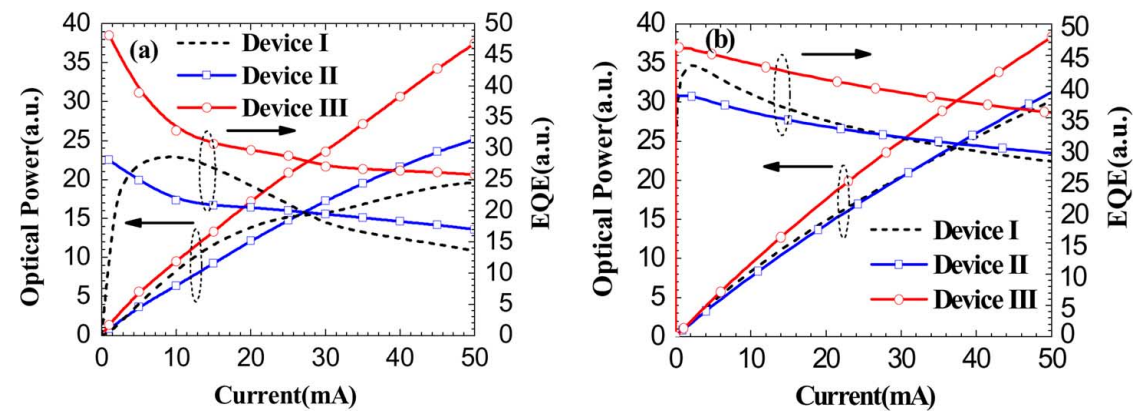

Fig. 2. (a) Experimentally measured and (b) numerically simulated optical output power and EQE as a function of current for Devices I, II, and III.

by Si-doped quantum barriers) could screen the quantum confined Stark effect (QCSE), though the screening effect is not optimum due to the absence of ionized dopants [35]. In this work, we study both numerically and experimentally on the step-doping of the quantum barriers with $\mathrm{Si}$, which could effectively screen the QCSE through the ionized dopants by properly designing the doped thickness and position in the quantum barriers. This provides additional degree of freedom of designing thicker quantum wells to avoid carrier high energy state filling, relieving the efficiency droop in c-plane LEDs [36]. The proposed step-doped quantum barriers could reduce hole blocking effect, promote electron injection, quench polarization fields and enhance electron-hole wave function overlap $\left(\Gamma_{\mathrm{e}-\mathrm{hh}}\right)$ within the quantum wells. These improvements translate to the enhancement of optical output power and efficiency.

\section{EXPERIMENTS}

To investigate the proposed step-doped barriers, $\mathrm{InGaN} / \mathrm{GaN}$ LED epitaxial wafers were grown by AIXTRON close-coupled showerhead metal-organic chemical-vapor deposition (MOCVD) reactor on c-plane sapphire substrates [37]. The growth was initiated on a $30 \mathrm{~nm}$ thick low-temperature grown $\mathrm{u}-\mathrm{GaN}$ buffer layer (at $560{ }^{\circ} \mathrm{C}$ with a growth pressure of 600 mbar and a V/III ratio between $\mathrm{NH}_{3}$ and TMGa of 950). A $2 \mu \mathrm{m}$ thick u-GaN layer was subsequently grown at 1050 ${ }^{\circ} \mathrm{C}$ with a growth pressure of $400 \mathrm{mbar}$ and a V/III ratio of 2700. For the n-GaN growth (with $N_{D}=8 \times 10^{18} \mathrm{~cm}^{-3}$ ), the growth temperature, pressure and V/III ratio were set to $1060{ }^{\circ} \mathrm{C}, 250$ mbar and 1140 , respectively. A higher V/III ratio of 10064 was utilized for the growth of quantum barriers. The growth temperature was $820^{\circ} \mathrm{C}$ and $737^{\circ} \mathrm{C}$ for the quantum barriers and quantum wells, respectively, while the $\mathrm{V} / \mathrm{III}([\mathrm{NH} 3] /[\mathrm{TEGa}]+[\mathrm{TMIn}])$ ratio during the quantum well growth was 10500 . However, a constant growth pressure of 400 mbar was used during the growth of both the quantum barriers and quantum wells. The LED samples were finally covered with a $300 \mathrm{~nm}$ thick $\mathrm{p}-\mathrm{GaN}$ grown at $950^{\circ} \mathrm{C}$ with the pressure of $150 \mathrm{mbar}$, and the hole concentration of our $\mathrm{p}-\mathrm{GN}$ is $1.0 \times 10^{17} \mathrm{~cm}^{-3}$. The structures were annealed in the ambient of $\mathrm{N}_{2}$ for $15 \mathrm{~min}$ at $687{ }^{\circ} \mathrm{C}$. In our experiment, $\mathrm{Cp}_{2} \mathrm{Mg}$ and diluted $\mathrm{SiH}_{4}$ were used as p-type and n-type dopant sources, respectively.

In our study, we comparatively studied three structures of InGaN/GaN LED epi-wafers, which are called Devices I, II, and III. The schematic diagrams of the investigated devices are shown in Fig. 1. Among them, Device I is a standard LED with undoped barriers, while Device II is designed with $12 \mathrm{~nm}$ thick barriers each fully doped with $\operatorname{Si}\left(N_{D}=2.6 \times 10^{18} \mathrm{~cm}^{-3}\right)$, and Device III features step-doped barriers (6 $\mathrm{nm}$ undoped and followed by $6 \mathrm{~nm}$ doped with $\left.N_{D}=2.6 \times 10^{18} \mathrm{~cm}^{-3}\right)$. The three devices differ only in their quantum barriers.

The studied LEDs all consist of 5-pair quantum well stack $\left(\operatorname{In}_{0.15} \mathrm{Ga}_{0.85} \mathrm{~N} / \mathrm{GaN}\right.$ with $3 \mathrm{~nm}$ well and $12 \mathrm{~nm}$ barrier$)$ as the active region. The devices were fabricated by using standard fabrication process. The LED mesa was obtained through reactive ion etch with a size of $300 \mu \mathrm{m} \times 300 \mu \mathrm{m}$. Ni/Au $(5 \mathrm{~nm} / 150$ $\mathrm{nm}$ ) was deposited as the p-electrode, and then the thermal annealing was performed for the p-electrode in the mixture of $\mathrm{N}_{2}$ and $\mathrm{O}_{2}$ for $5 \mathrm{~min}$. Finally Ti/Au $(30 \mathrm{~nm} / 150 \mathrm{~nm})$ was deposited on the $\mathrm{n}$-GaN layer as the $\mathrm{n}$-electrode.

\section{RESULTS AND DISCUSSION}

Fig. 2(a) shows the measured EQE and optical output power as a function of the current for all the devices (along with Fig. 2(b) depicting the numerical simulation results). As demonstrated, Device II performs better than Device I when the current is increased beyond $26.5 \mathrm{~mA}$, as the Si-doped barriers replenish electrons in the quantum wells. Furthermore, the screening effect on the QCSE improves the spatial overlap 

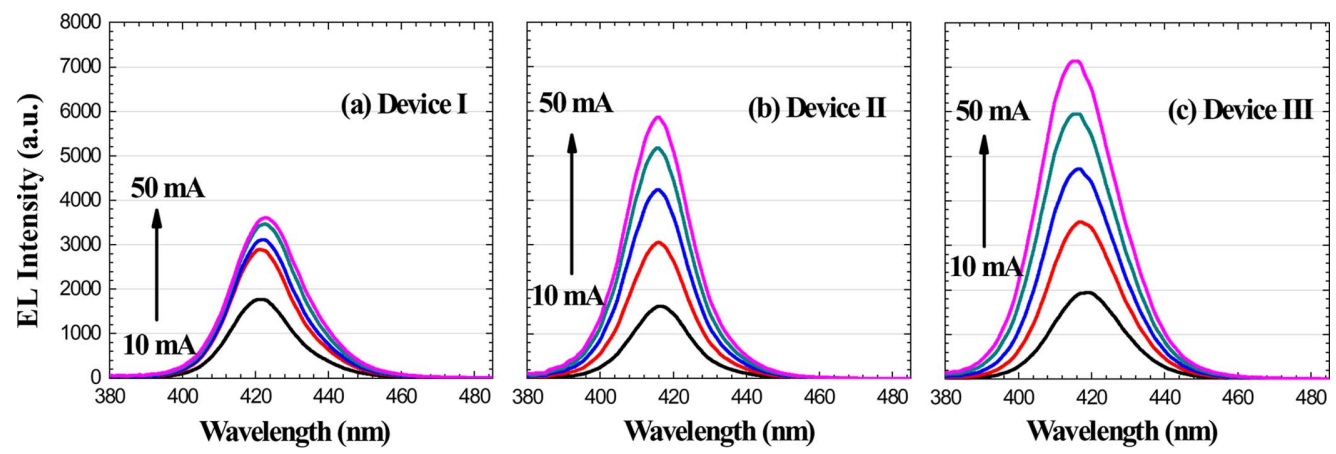

Fig. 3. EL spectra for: (a) Device I; (b) Device II; and (c) Device III.
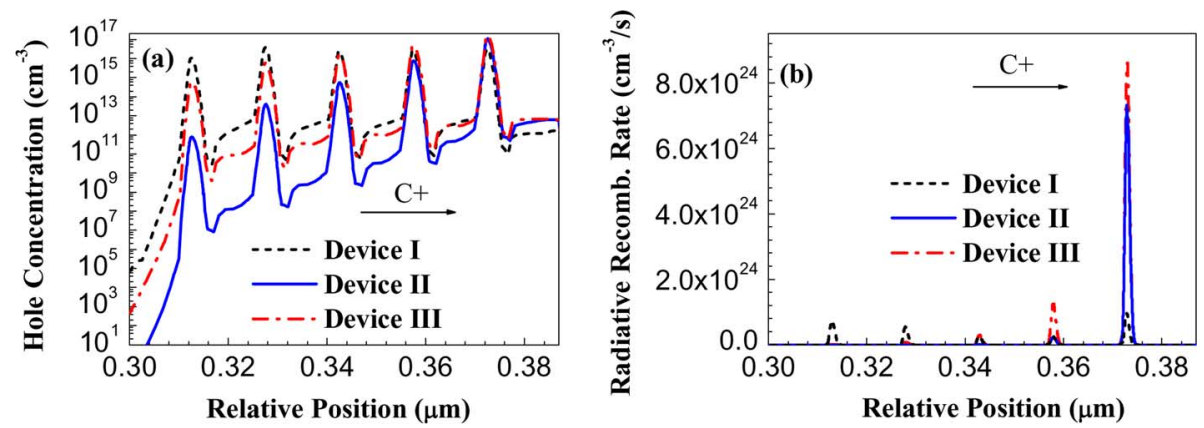

Fig. 4. (a) Simulated hole concentration and (b) simulated radiative recombination rates for Devices I, II, and III at I $=50 \mathrm{~mA}$.

between the electron and hole wave functions [29], thus enhancing the radiative recombination rates. Nevertheless, in the low current regime $(4.8 \mathrm{~mA}<\mathrm{I}<26.5 \mathrm{~mA})$, Device II performs worse than Device I. On the other hand, across the whole current range tested, we see that Device III outperforms Devices I and II, and the power is experimentally enhanced by $90.79 \%$ between Devices I and III, while $27.90 \%$ between Devices I and III at $50 \mathrm{~mA}$. Fig. 3 presents the electroluminescence (EL) for the studied devices, where the emission intensity is the strongest for Device III and the weakest for Device I. Meanwhile, Devices II and III show a shorter peak emission wavelength compared to Device I, which is attributed to the slightly relieved QCSE by Si-doped quantum barriers [29]. However, the less pronounced blue-shift for all the three devices as the injection current increases is caused by the junction heating effect [38].

In order to better understand the improvement of EQE and optical output power in Devices I, II and III, numerical simulations were performed by APSYS [39], which self-consistently solves the Poisson equation, continuity equation and Schrödinger equation with proper boundary conditions. The self-consistent six-band $k \cdot p$ theory is used to take account of the effect of carrier screening in InGaN quantum wells [40]. Here, the Auger recombination coefficients are taken to be $1 \times 10^{-30}$ $\mathrm{cm}^{6} / \mathrm{s}$ [7], [41]. The offset ratio between the conduction and valence bands for $\mathrm{InGaN} / \mathrm{GaN}$ quantum well is assumed to be $70: 30$ [42]. Also, a $40 \%$ of the theoretical polarization charge is used due to the crystal relaxation through generating dislocations [43]. The other parameters used in the simulation can be found elsewhere [44]. Fig. 2(b) depicts the calculated EQE and optical output power, which demonstrates that similar trends of the enhanced EQE and optical output power are observed after employing Si-doped quantum barriers in Device III compared to Devices I and II. However, in the simulation, we did not consider the localized states caused by potential fluctuation of InGaN alloys [45], and the temperature/carrier concentration dependence of those non-radiative recombination factors (e.g., Auger recombination, Shockley-Read-Hall recombination), which caused the discrepancy between simulation and experiment [refer to Fig. 2(a) and (b)].

It is reported that the wholly-doped barrier increases the barrier height for holes, thus retarding the hole injection [32], which explains the worse performance of Device II compared to Device I in low current regime $(4.8 \mathrm{~mA}<\mathrm{I}<26.5 \mathrm{~mA})$. Fortunately, hole-blocking effects in Device II can be suppressed by employing step-doped barriers. It is observed from Fig. 2(a) and (b) that Device III performs better than Device II due to an improved hole transport.

To better probe the hole transport of Devices I, II, and III, we simulated the hole distribution and radiative recombination rates in their quantum wells [Fig. 4(a) and (b)]. As shown in Fig. 4(a), all devices possess the highest hole concentration in the fifth quantum well (the one closest to $\mathrm{p}-\mathrm{GaN}$ ) along [0001]orientation. However, due to the highest valence-band barrier height in the wholly-doped Si barriers (Fig. 5 and Table I), Device II cannot inject holes efficiently into the quantum wells that are close to $\mathrm{n}-\mathrm{GaN}$ side (e.g., the first quantum well). On the other hand, Device III has half the thickness of doped barriers compared to Device II, which reduces the overall valence-band barrier height for the hole injection (Fig. 5 and Table I). As a result, a much more homogeneous hole distribution can be obtained in Device III, which correspondingly leads to higher radiative recombination rates in the quantum wells close to the $\mathrm{n}-\mathrm{GaN}$ side for Device III compared to Device II. Even though 


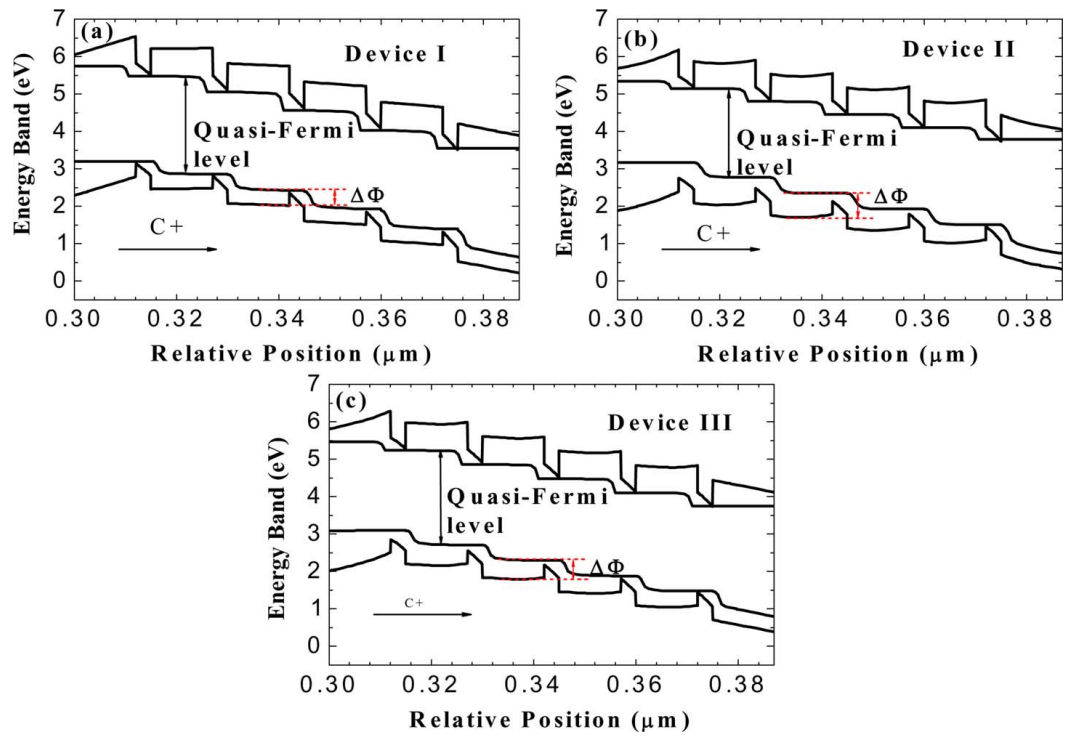

Fig. 5. (a) Simulated energy band for (a) Device I, (b) Device II, and (c) Device III. $\Delta \Phi$ represents the energy barrier height for holes.

TABLE I

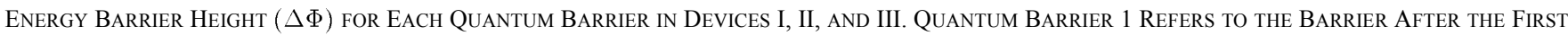

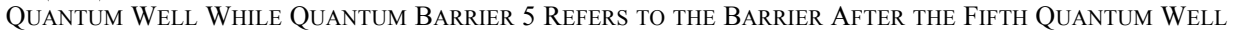

\begin{tabular}{lccc}
\hline & Device I $(\boldsymbol{\Delta} \boldsymbol{\Phi})$ & Device II $(\boldsymbol{\Delta} \boldsymbol{\Phi})$ & Device III $(\boldsymbol{\Delta} \boldsymbol{\Phi})$ \\
\hline Quantum Barrier 1 & $402 \mathrm{meV}$ & $750 \mathrm{meV}$ & $563 \mathrm{meV}$ \\
Quantum Barrier 2 & $402 \mathrm{meV}$ & $646 \mathrm{meV}$ & $510 \mathrm{meV}$ \\
Quantum Barrier 3 & $410 \mathrm{meV}$ & $580 \mathrm{meV}$ & $479 \mathrm{meV}$ \\
Quantum Barrier 4 & $410 \mathrm{meV}$ & $500 \mathrm{meV}$ & $455 \mathrm{meV}$ \\
Quantum Barrier 5 & $406 \mathrm{meV}$ & $415 \mathrm{meV}$ & $413 \mathrm{meV}$ \\
\hline
\end{tabular}

Device I shows the most homogeneous hole distribution among the three devices due to the smallest energy barrier height (Fig. 5 and Table I), it suffers from the strongest QCSE and hence the low radiative recombination rates, as shown in Fig. 4(b).

As is well recognized, the strong polarization induced field within the quantum wells spatially separates the electron and hole wave functions, thus reducing the interband transition probability of carriers. However, the internal electric field profile can be tuned by Si-doping the quantum barriers. On the other hand, it can be seen clearly from Fig. 4(b) that the fifth quantum well dominates the radiative recombination rates especially for Devices II and III. Thus analyzing the electric field in the fifth quantum well for these three devices comparatively is helpful for us to understand the mechanism for the QCSE suppression. Fig. 6(a) presents the electric field in the fifth quantum well for Devices I, II, and III under equilibrium, where the positive direction is along [0001]. We can see a considerably flat electric field profile in Device I, whereas for devices with Si-doped quantum barriers (Devices II and III), the electric field is tilted as depicted in Fig. 6(a); a reduction of the electric field in the well close to the n-GaN side ["B" site in Fig. 6(a)] is achieved, while an enhanced magnitude of electric field is simultaneously triggered at the interface close to p-GaN side ["A" site in Fig. 6(a)]. Fig. 6(b)-(d) shows the energy band diagrams and the charge profile for Devices I, II, and III under equilibrium, respectively. In Device I, only polarization induced charges are shown in Fig. 6(b), since there are no Si dopants in the quantum barriers and the simulated electron sheet charge density $\left(n_{S}\right)$ in the fifth quantum well is around $1.4 \times 10^{2} \mathrm{~cm}^{-2}$, which is negligible compared to the polarization charge density that is in the order of $10^{13} \mathrm{~cm}^{-2}$ [29]. Thus we obtain the macroscopic electric field in (1) at both " $\mathrm{A}$ " and "B" sites, respectively, which explains the field symmetry for Device I in Fig. 6(a)

$$
\left|\vec{E}_{s p+p z}\right|=\frac{e \cdot \sigma_{S}}{\varepsilon_{r} \cdot \varepsilon_{s}}
$$

where $e$ is the elementary electronic charge, $\varepsilon_{r}$ is the relative dielectric constant of InGaN, $\varepsilon_{s}$ is the it electric permittivity in vacuum, and $\sigma_{S}$ is denoted as the polarization induced charge density.

For Devices II and III, the Si dopants can be considered to be completely ionized [46], feeding electrons into the quantum well and leaving a depletion region in the barrier. The sheet charge density of the ionized $\mathrm{Si}$ atoms can be obtained from $\sigma_{S i}=N_{D} \cdot t_{D}$, where $N_{D}$ is the Si dopant concentration $\left(N_{D}=\right.$ $2.6 \times 10^{18} \mathrm{~cm}^{-3}$ for both Devices II and III) and $t_{D}$ is the doped barrier thickness $\left(t_{D}=12 \mathrm{~nm}\right.$ and $6 \mathrm{~nm}$ in for Devices II and III, respectively), and therefore we obtain the sheet charge density of $\mathrm{Si}\left(\sigma_{\mathrm{Si}}\right)$, which is $3.12 \times 10^{12} \mathrm{~cm}^{-2}$ and $1.56 \times 10^{12} \mathrm{~cm}^{-2}$ for Device II and III, respectively. Besides, according to our simulation, the $n_{S}$ in the fifth quantum well is about $3.0 \times 10^{12}$ $\mathrm{cm}^{-2}$ and $1.5 \times 10^{12} \mathrm{~cm}^{-2}$ in Devices II and III, respectively, 

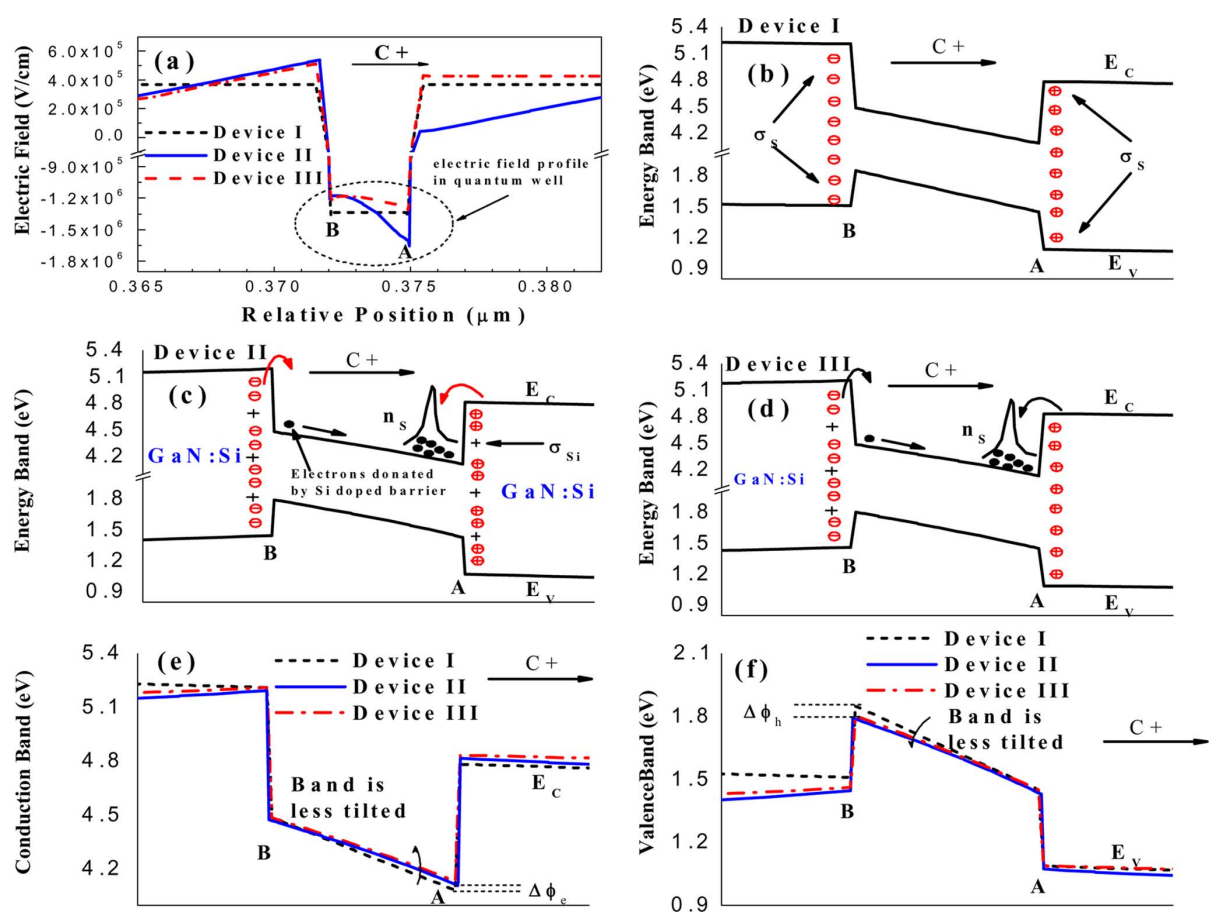

Fig. 6. (a) Simulated electric field profile in the fifth quantum well, where the positive direction is along the [0001], energy band diagram and charge profile for: (b) Device I; (c) Device II; (d) Device III; (e) combined conduction band diagrams; and (f) combined valance band diagrams for Devices I, II, and III. Data collected under equilibrium.

which are slightly smaller than $\sigma_{\mathrm{Si}}$ by our simple calculation above. The smaller $n_{S}$ compared to $\sigma_{S i}$ is due to the loss of electron leaking into $\mathrm{p}-\mathrm{GaN}$ region. Since we do not observe any holes diffusing into the quantum wells under the equilibrium state in the simulations, the effect of holes is not included here.

Accordingly, the electric field at "A" site in Device II can be given by (2) [refer to Fig. 6(c)], while it can be expressed in (3) for Device III if the diffused $\sigma_{\mathrm{Si}}$ from the doped part in the quantum barrier is negligible compared to $\sigma_{S}$ as shown in Fig. 6(d). However, the electric field at "B" site for both Devices II and III can be represented in (4) according to Fig. 6(c) and (d)

$$
\begin{aligned}
& \left|\vec{E}_{s p+p z}+\vec{E}_{S i}+\vec{E}_{n_{S}}\right|=\frac{e \cdot\left|\left(\sigma_{S}+\sigma_{S i}-n_{S}\right)\right|}{\varepsilon_{r} \cdot \varepsilon_{s}} \\
& \left|\vec{E}_{s p+p z}+\vec{E}_{n_{S}}\right|=\frac{e \cdot\left|\left(\sigma_{S}-n_{S}\right)\right|}{\varepsilon_{r} \cdot \varepsilon_{s}} \\
& \left|\vec{E}_{s p+p z}+\vec{E}_{S i}\right|=\frac{e \cdot\left|\left(\sigma_{S}-\sigma_{S i}\right)\right|}{\varepsilon_{r} \cdot \varepsilon_{s}}
\end{aligned}
$$

where $\vec{E}_{S i}$ and $\vec{E}_{n_{S}}$ represent the electric field caused by Si dopants and electrons, respectively.

It is well-known that the idea to screen the QCSE by introducing $\mathrm{Si}$ dopants in the quantum barriers is realized by releasing electrons [33] into the quantum wells [i.e., $n_{S}$ in (2) and (3)], but, the effect of the ionized donors has never been properly recognized. As shown in (4), a reduced electric field at "B" site [Fig. 6(a)] caused by the presence of ionized Si dopants [Fig. 6(c) and (d)] helps to make the valence band less titled for Devices II and III compared to Device I (Fig. 6(f), $\Delta \phi_{h}=60$ $\mathrm{meV}$ for Device II and $50 \mathrm{meV}$ for Device III), which in turn pushes the hole wave function towards "A" site [Fig. 7(a)]. Thus, Device II and III enjoy a more overlapped electron-hole wave function than Device I. Device I has a $\Gamma_{\mathrm{e}-\mathrm{hh}}$ of $29.94 \%$, while Device II and Device III feature a $\Gamma_{\mathrm{e}-\mathrm{hh}}$ of $34.81 \%$ and $37.76 \%$, respectively. The smallest $\Gamma_{\mathrm{e}-\mathrm{hh}}$ is responsible for the weakest emission intensity for Device I [Fig. 3(a)]. Moreover, the more increased $\Gamma_{\mathrm{e}-\mathrm{hh}}$ in Device III compared to Device II is attributed to the reduced electric field at "A" site compared to Device II, as a reduced field at "A" site that is caused by the absence of ionized Si dopants [refer to (2) and (3)] tilts the conduction band more (Fig. 6(e), $\Delta \phi_{e}=26 \mathrm{meV}$ for Device II and $50 \mathrm{meV}$ for Device III) and pushes the electron wave function towards "B" site more [Fig. 7(a)]. Therefore, the largest $\Gamma_{\mathrm{e}-\mathrm{hh}}$ translates to the strongest emission intensity for Device III as shown in Fig. 3(c). Moreover, according to our simulation, Device III shows even better screening effect to QCSE than Device II, and thus ideally a shorter wavelength is expected for Device III. However, as shown in Fig. 3(b) and (c), the peak emission wavelengths for Devices II and III are very close, the difference is ranged from 1.9 to $0.4 \mathrm{~nm}$ for various currents we used. As the devices were grown in different runs by MOCVD, it is possible, for example, we may have some slight difference in Indium incorporation in the quantum wells. The peak wavelength difference between Device II and III is a combined result generated from different QCSE screening effect and possibly Indium incorporation. Nevertheless, in order to verify the effect of step-doping feature, we have further investigated $\Gamma_{\mathrm{e}-\mathrm{hh}}$ for Devices II and III as a function of Si doping concentration in their quantum barriers as shown in Fig. 7(b), which indicates the advantage of the step-doped architecture in Device III over the wholly doped barriers in Device II.

\section{CONCLUSION}

In conclusion, the effect of Si step-doped quantum barriers on the optical power and EQE of InGaN/GaN LEDs is studied. 

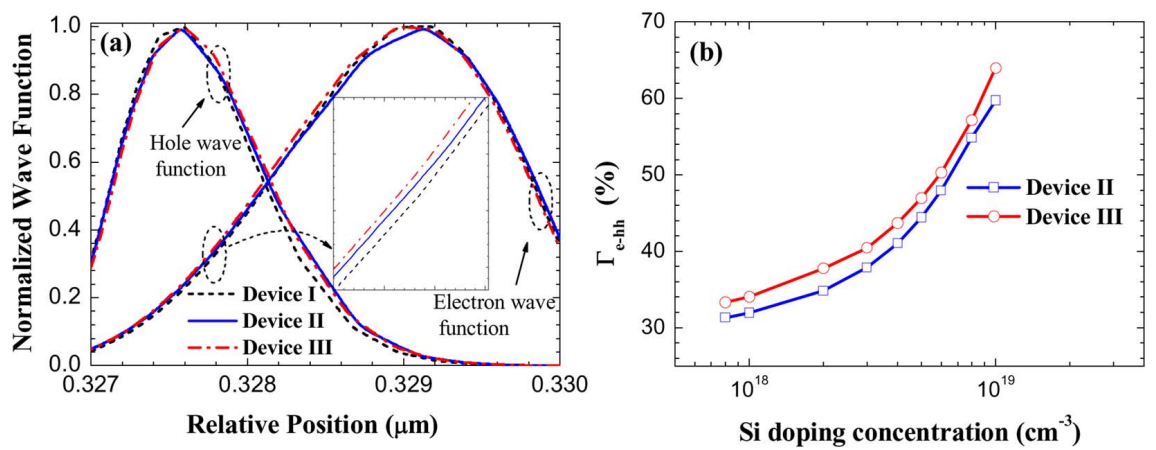

Fig. 7. (a) Normalized electron and hole wave functions for Devices I, II, and III at $50 \mathrm{~mA}$, and (b) $\Gamma_{\mathrm{e}-\mathrm{hh}}$ for Devices II and III as a function of Si-doping concentration at $50 \mathrm{~mA}$.

Improvements have been observed in the proposed LED device with Si step-doped quantum barriers. This is mainly attributed to the reduced barrier height for the hole injection and the excellent screening effect on QCSE. Furthermore, LEDs with Si step-doped quantum barriers shows better screening effect on the QCSE than LEDs with Si fully doped quantum barriers. The proposed approach of step-doped quantum barriers can be used to increase the efficiency and hence holds great promise for high-efficiency GaN-based LEDs.

\section{ACKNOWLEDGMENT}

The authors would like to thank Dr. K. L. Ke and Dr. C. B. Soh for their assistance in electroluminescence measurement in IMRE (Institute of Materials Research and Engineering) of A*STAR (Agency for Science, Technology and Research).

\section{REFERENCES}

[1] S. Nakamura, M. Senoh, and T. Mukai, "P-GaN/N-InGaN/N-GaN double-heterostructure blue-light-emitting diodes," Jpn. J. Appl.Phys. Pt. 2-Lett., vol. 32, pp. L8-L11, Jan. 1993.

[2] S. T. Tan, X. W. Sun, H. V. Demir, and S. P. DenBaars, "Advances in the LED materials and architectures for energy-saving solid-state lighting toward 'lighting revolution'," IEEE Photon. J., vol. 4, no. 2, pp. 613-619, Apr. 2012.

[3] A. A. Efremov, N. I. Bochkareva, R. I. Gorbunov, D. A. Lavrinovich, Y. T. Rebane, D. V. Tarkhin, and Y. G. Shreter, "Effect of the Joule heating on the quantum efficiency and choice of thermal conditions for high-power blue InGaN/GaN LEDs," Semiconductors, vol. 40, pp. 605-610, May 2006.

[4] M. H. Kim, M. F. Schubert, Q. Dai, J. K. Kim, E. F. Schubert, J. Piprek, and Y. Park, "Origin of efficiency droop in GaN-based light-emitting diodes," Appl. Phys. Lett., vol. 91, pp. 183507-1-183507-3, Oct. 2007.

[5] W. Chow, M. H. Crawford, J. Y. Tsao, and M. Kneissl, "Internal efficiency of InGaN light-emitting diodes: Beyond a quasiequilibrium model," Appl. Phys. Lett., vol. 97, pp. 121105-1-121105-3, Sep. 2010.

[6] H. Y. Ryu and J. I. Shim, "Effect of current spreading on the efficiency droop of InGaN light-emitting diodes," Opt. Express, vol. 19, pp. 2886-2894, Feb. 2011.

[7] Y. C. Shen, G. O. Mueller, S. Watanabe, N. F. Gardner, A. Munkholm, and M. R. Krames, "Auger recombination in InGaN measured by photoluminescence," Appl. Phys. Lett., vol. 91, pp. 141101-1-141101-3, Oct. 2007.

[8] Y. J. Lee, C. H. Chen, and C. J. Lee, "Reduction in the efficiency-droop effect of InGaN green light-emitting diodes using gradual quantum wells," IEEE Photon. Technol. Lett., vol. 22, no. 10, pp. 1506-1508, Oct. 2010
[9] C. H. Wang, S. P. Chang, W. T. Chang, J. C. Li, Y. S. Lu, Z. Y. Li, H. C. Yang, H. C. Kuo, T. C. Lu, and S. C. Wang, "Efficiency droop alleviation in InGaN/GaN light-emitting diodes by graded-thickness multiple quantum wells," Appl. Phys. Lett., vol. 97, pp. 181101-1-181101-3, Nov. 2010.

[10] H. P. Zhao, G. Y. Liu, R. A. Arif, and N. Tansu, "Current injection efficiency induced efficiency-droop in InGaN quantum well light-emitting diodes," Solid-State Electron., vol. 54, pp. 1119-1124, Oct. 2010.

[11] S. J. Huang, Y. K. Su, C. Y. Tseng, S. C. Lin, and H. C. Hsu, "Improvement of light intensity for nitride-based multi-quantum well light emitting diodes by stepwise-stage electron emitting layer," Appl. Phys. Express, vol. 3, pp. 122106-1-122106-3, 2010.

[12] S. J. Lee, S. H. Han, C. Y. Cho, S. P. Lee, D. Y. Noh, H. W. Shim, Y. C. Kim, and S. J. Park, "Improvement of GaN-based light-emitting diodes using p-type $\mathrm{AlGaN} / \mathrm{GaN}$ superlattices with a graded $\mathrm{Al}$ composition," J. Phys. D-Appl. Phys., vol. 44, p. 105101, Mar. 2011.

[13] Y. K. Kuo, J. Y. Chang, and M. C. Tsai, "Enhancement in hole-injection efficiency of blue InGaN light-emitting diodes from reduced polarization by some specific designs for the electron blocking layer," Opt. Lett., vol. 35, pp. 3285-3287, Oct. 2010.

[14] S. Choi, H. J. Kim, S. S. Kim, J. Liu, J. Kim, J. H. Ryou, R. D. Dupuis, A. M. Fischer, and F. A. Ponce, "Improvement of peak quantum efficiency and efficiency droop in III-nitride visible light-emitting diodes with an InAlN electron-blocking layer," Appl. Phys. Lett., vol. 96, pp. 221105-1-221105-3, May 2010.

[15] J. Simon, V. Protasenko, C. X. Lian, H. L. Xing, and D. Jena, "Polarization-induced hole doping in wide-band-gap uniaxial semiconductor heterostructures," Science, vol. 327, pp. 60-64, Jan. 2010.

[16] R. A. Arif, Y. K. Ee, and N. Tansu, "Polarization engineering via staggered InGaN quantum wells for radiative efficiency enhancement of light emitting diodes," Appl. Phys. Lett., vol. 91, pp. 091110-1-091110-3, Aug. 2007.

[17] R. A. Arif, H. P. Zhao, Y. K. Ee, and N. Tansu, "Spontaneous emission and characteristics of staggered InGaN quantum-well light-emitting diodes," IEEE J. Quantum Electron., vol. 44, pp. 573-580, May-Jun. 2008.

[18] H. P. Zhao, G. Y. Liu, J. Zhang, J. D. Poplawsky, V. Dierolf, and N. Tansu, "Approaches for high internal quantum efficiency green InGaN light-emitting diodes with large overlap quantum wells," Opt. Express, vol. 19, pp. A991-A1007, Jul. 2011.

[19] H. P. Zhao, G. Y. Liu, X. H. Li, G. S. Huang, J. D. Poplawsky, S. T. Penn, V. Dierolf, and N. Tansu, "Growths of staggered InGaN quantum wells light-emitting diodes emitting at 520-525 nm employing graded growth-temperature profile," Appl. Phys. Lett., vol. 95, pp. 0611041-061104-3, Aug. 2009.

[20] H. P. Zhao and N. Tansu, "Optical gain characteristics of staggered InGaN quantum wells lasers," J. Appl. Phys., vol. 107, pp. 1131101-113110-12, Jun. 2010.

[21] J. Zhang and N. Tansu, "Improvement in spontaneous emission rates for InGaN quantum wells on ternary InGaN substrate for light-emitting diodes," J. Appl. Phys., vol. 110, pp. 113110-1-113110-5, Dec. 2011.

[22] J. H. Son and J. L. Lee, "Strain engineering for the solution of efficiency droop in InGaN/GaN light-emitting diodes," Opt. Express, vol. 18, pp. 5466-5471, Mar. 2010.

[23] H. P. Zhao, R. A. Arif, and N. Tansu, "Self-consistent gain analysis of type-II 'W' InGaN-GaNAs quantum well lasers," J. Appl. Phys., vol. 104, pp. 043104-1-043104-7, Aug. 2008. 
[24] H. P. Zhao, G. Y. Liu, and N. Tansu, "Analysis of InGaN-delta-InN quantum wells for light-emitting diodes," Appl. Phys. Lett., vol. 97, pp. 131114-1-131114-3, Sep. 2010.

[25] S. H. Park, D. Ahn, B. H. Koo, and J. E. Oh, "Optical gain improvement in type-II InGaN/GaNSb/GaN quantum well structures composed of InGaN/and GaNSb layers," Appl. Phys. Lett., vol. 96, pp. 0511061-051106-3, Feb. 2010.

[26] J. Zhang, H. P. Zhao, and N. Tansu, "Large optical gain AlGaNdelta-GaN quantum wells laser active regions in mid-and deep-ultraviolet spectral regimes," Appl. Phys. Lett., vol. 98, pp. 1711111-171111-3, Apr. 2011.

[27] R. M. Farrell, P. S. Hsu, D. A. Haeger, K. Fujito, S. P. Denbaars, J. S. Speck, and S. Nakamura, "Low-threshold-current-density AlGaNcladding-free $\mathrm{m}$-plane InGaN/GaN laser diodes," Appl. Phys. Lett., vol. 96, pp. 231113-1-231113-3, 2010.

[28] R. M. Farrell, D. A. Haeger, P. S. Hsu, K. Fujito, D. F. Feezell, S. P. Denbaars, J. S. Speck, and S. Nakamura, "Determination of internal parameters for AlGaN-cladding-free m-plane InGaN/GaN laser diodes," Appl. Phys. Lett., vol. 99, pp. 171115-1-171115-3, 2011.

[29] L. W. Wu, S. J. Chang, T. C. Wen, Y. K. Su, J. F. Chen, W. C. Lai, C. H. Kuo, C. H. Chen, and J. K. Sheu, "Influence of Si-doping on the characteristics of InGaN-GaN multiple quantum-well blue light emitting diodes," IEEE J. Quantum Electron., vol. 38, no. 5, pp. 446-450, May 2002.

[30] Z. Zheng, Z. Chen, Y. Xian, B. Fan, S. Huang, W. Jia, Z. Wu, G. Wang, and H. Jiang, "Enhanced electrostatic discharge properties of nitridebased light-emitting diodes with inserting Si-delta-doped layers," Appl. Phys. Lett., vol. 99, pp. 111109-1-111109-3, 2011.

[31] H. P. D. Schenk, A. Bavard, E. Frayssinet, X. Song, F. Cayrel, H Ghouli, M. Lijadi, L. Naïm, M. Kennard, Y. Cordier, D. Rondi, and D. Alquier, "Delta-doping of epitaxial GaN layers on large diameter Si(111) substrates," Appl. Phys. Express, vol. 5, pp. 025504-1-025504-3, 2012.

[32] J. H. Ryou, J. Limb, W. Lee, J. P. Liu, Z. Lochner, D. W. Yoo, and R. D. Dupuis, "Effect of silicon doping in the quantum-well barriers on the electrical and optical properties of visible green light-emitting diodes," IEEE Photon. Technol. Lett., vol. 20, no. 11, pp. 1769-1771, Nov. 2008.

[33] M. K. Kwon, K. Park, S. H. Baek, J. Y. Kim, and S. J. Park, "Si delta doping in a $\mathrm{GaN}$ barrier layer of InGaN/GaN multiquantum well for an efficient ultraviolet light-emitting diode," Journal of Appl. Phys., vol. 97, pp. 106109-1-106109-3, May 2005.

[34] D. Zhu, A. N. Noemaun, M. F. Schubert, J. Cho, E. F. Schubert, M. H. Crawford, and D. D. Koleske, "Enhanced electron capture and symmetrized carrier distribution in GaInN light-emitting diodes having tailored barrier doping," Appl. Phys. Lett., vol. 96, pp. 121110-1-121110-3, Mar. 2010.

[35] V. Fiorentini, F. Bernardini, F. Della Sala, A. Di Carlo, and P. Lugli, "Effects of macroscopic polarization in III-V nitride multiple quantum wells," Phys. Rev. B, vol. 60, pp. 8849-8858, Sep. 1999.

[36] L. Wang, C. M. Lu, J. N. Lu, L. Liu, N. Y. Liu, Y. J. Chen, Y. F. Zhang, E. D. Gu, and X. D. Hu, "Influence of carrier screening and band filling effects on efficiency droop of InGaN light emitting diodes," Opt. Express, vol. 19, pp. 14182-14187, July 2011.

[37] Z. G. Ju, S. T. Tan, Z.-H. Zhang, Y. Ji, Z. Kyaw, Y. Dikme, X. W Sun, and H. V. Demir, "On the origin of the redshift in the emission wavelength of InGaN/GaN blue light emitting diodes grown with a higher temperature interlayer," Appl. Phys. Lett., vol. 100, pp. 123503 1-123503-3, 2012.

[38] Z. Gong, S. Jin, Y. Chen, J. McKendry, D. Massoubre, I. M. Watson, E. Gu, and M. D. Dawson, "Size-dependent light output, spectral shift, and self-heating of $400 \mathrm{~nm}$ InGaN light-emitting diodes," J. Appl. Phys., vol. 107, pp. 013103-1-013103-6, 2010.

[39] APSYS by Crosslight Software Inc., Burnaby, Canada, [Online]. Available: http://www.crosslight.com

[40] H. Zhao, R. A. Arif, Y. K. Ee, and N. Tansu, "Self-cnsistent analysis of strain-compensated InGaN-AlGaN quantum wells for lasers and lightemitting diodes," IEEE J. Quantum Electron., vol. 45, pp. 66-78, 2009.

[41] K. T. Delaney, P. Rinke, and C. G. Van De Walle, "Auger recombination rates in nitrides from first principles," Appl. Phys. Lett., vol. 94, pp. 191109-1-191109-3, 2009.

[42] J. Piprek, "Efficiency droop in nitride-based light-emitting diodes," Phys. Status Solid. a-Appl. Mater. Sci., vol. 207, pp. 2217-2225, Oct. 2010 .

[43] V. Fiorentini, F. Bernardini, and O. Ambacher, "Evidence for nonlinear macroscopic polarization in III-V nitride alloy heterostructures," Appl. Phys. Lett., vol. 80, pp. 1204-1206, Feb. 2002.
[44] I. Vurgaftman and J. R. Meyer, "Band parameters for nitrogen-containing semiconductors," J. Appl. Phys., vol. 94, pp. 3675-3696, Sep. 2003.

[45] T. Deguchi, A. Shikanai, K. Torii, T. Sota, S. Chichibu, and S. Nakamura, "Luminescence spectra from InGaN multiquantum wells heavily doped with Si," Appl. Phys. Lett., vol. 72, pp. 3329-3331, Jun. 1998.

[46] L. T. Romano, C. G. Van de Walle, J. W. Ager, W. Gotz, and R. S. Kern, "Effect of Si doping on strain, cracking, and microstructure in GaN thin films grown by metalorganic chemical vapor deposition," $J$. Appl. Phys., vol. 87, pp. 7745-7752, Jun. 2000.

Zi-Hui Zhang received the B.S. degree from the School of Physics, Shandong University, China, in 2006, and is currently working toward the Ph.D. degree in the School Electrical and Electronics Engineering, Nanyang Technological University, Singapore.

His research interests are in the epitaxy growth, characterization and fabrication of III-nitride optoelectronic devices by Metal-organic Chemical-vapor Deposition. He is also focused on the modeling and simulation of III-nitride light emitting devices.

Swee Tiam Tan received the B.Eng. and Ph.D. degrees from Nanyang Technological University, Singapore, in 2003 and 2007, respectively.

From 2007 to 2010, he was with the Semiconductor Process Technologies Laboratory, Institute of Microelectronics, A*STAR, Singapore, where he worked on $\mathrm{ZnO}$ epitaxial growth by metal-organic chemical-vapor deposition. Since 2010, he has been with the Nanyang Technological University, Singapore, where he is currently the Program Manager for LUMINOUS! Center of Excellence for Semiconductor Lighting and Displays. He has authored or coauthored more than 50 international referred journals and two book chapters. His current research interests include the epitaxial growth and characterization of semiconducting films, semiconductor LED lighting, OLED, OPV, and nanocrystal optoelectronics.

Zhengang Ju received the Ph.D. degree from Chinese Academy of Sciences, China, in 2009, and is currently a research fellow in LUMINOUS! Center of Excellence for Semiconductor Lighting and Displays of Nanyang Technological University. His research interests include MOCVD growth, fabrication and characterization of III-V and II-VI semiconductor devices.

Wei Liu received the Ph.D. degree in electrical engineering in National University of Singapore in 1999.

He is currently working with the School of Electrical and Electronic Engineering, Nanyang Technological University, Singapore. Before he joined Nanyang Technological University, he worked in Institute of Materials Research and Engineering, Agency of Science, Technology and Research, Singapore. His research interest includes semiconductor epitaxial growth, material characterization and semiconductor device design and fabrication. Currently, his research areas include group-III nitride LEDs, GaN power electronics and group-III nitride-based piezotronics and piezo-phototronics.

Yun Ji received the B.Eng. degree from Nanyang Technological University, Singapore, in 2009, and is currently working toward the Ph.D. degree at the School of Electrical and Electronics Engineering, Nanyang Technological University, Singapore.

His interests are focused on the epitaxy growth and device fabrication of GaNbased light-emitting diodes for lighting and display applications.

Zabu Kyaw received the B.Eng. from Nanyang Technological University, Singapore, in 2008, and is currently working towards to the Ph.D. degree at the School of Electrical and Electronics Engineering, Nanyang Technological University, Singapore.

His current research interests include the epitaxy growth and device fabrication of GaN based light emitting diodes. 
Yilmaz Dikme received the Ph.D. degree in electrical engineering from RWTH Aachen University, Germany, in 2006.

From 2006 till 2008, he worked for AIXTRON SE, Germany, as Project Manager for Advanced Process Support and after as consultant for the deposition of GaN and alloys. In 2010, he was the co-founder of AIXaTech GmbH, Aachen Germany, and as Technical Director of this company, he is additionally focused on the GaN growth, especially on large area silicon and sapphire wafer, also in the deposition of AlN layers by a low temperature process on silicon and sapphire to be used as AlN pseudo-substrates.

Xiao Wei Sun (SM'06) was born in Beijing, China, in 1968. He received the B.Eng., M.Eng, and Ph.D. degrees in photonics from Tianjin University, China, in 1990, 1992, and 1996 respectively, and also received the Ph.D. degree in electrical and electronic engineering from the Hong Kong University of Science and Technology, Hong Kong, in 1998.

In 1998, he joined the Division of Microelectronics in the School of Electrical and Electronic Engineering at Nanyang Technological University as an Assistant Professor. He was promoted to Associate Professor and Professor in 2005 and 2011, respectively. He has been working in the area of wide bandgap semiconductor materials and devices $(\mathrm{ZnO}$ and $\mathrm{GaN})$, organic electronics (organic light-emitting diodes, solar cells etc.), and liquid crystal photonic devices. $\mathrm{He}$ has (co-)authored more than 250 peer-reviewed journal publications in the area of photonics and microelectronics with more than 5000 external citations. His $\mathrm{H}$-index is 35 .

Dr. Sun is a Fellow of SPIE, Society for Information Display (SID), and Institute of Physics (IoP). He is the founder and Director of SID Singapore and Malaysia Chapter. He is the recipient of Nanyang Award for Research and Innovation 2009 for his contribution in $\mathrm{ZnO}$ nanodevices.
Hilmi Volkan Demir received the B.Sc. degree in electrical and electronics engineering from Bilkent University, Ankara, Turkey, in 1998, and the M.S. and $\mathrm{Ph} . \mathrm{D}$. degrees in electrical engineering from Stanford University, Stanford, CA, in 2000 and 2004, respectively.

In 2004, he joined Bilkent University as a faculty member and is an Associate Professor with joint appointments at the Department of Electrical and Electronics Engineering and the Department of Physics. In 2007, he received the Docent title (associate professorship) from the Turkish Council of Higher Education. In 2009, he has been awarded Singapore NRF Fellowship. $\mathrm{He}$ is concurrently Nanyang Associate Professor jointly with the School of Electrical and Electronic Engineering (Microelectronics Division) and the School of Physical and Mathematical Sciences (Physics and Applied Physics Division), Nanyang Technological University, Singapore, and the Director of the LUMINOUS! Center of Excellence for Semiconductor Lighting and Displays. His current research interests include the development of innovative devices and sensors, including the science and technology of excitonics for high-efficiency light generation and harvesting and wireless implant sensing for future healthcare. He has co-authored over $100 \mathrm{SCI}$ journal publications and delivered over 100 invited seminars and lectures in academia and industry.

Dr. Demir was the recipient of the European Science Foundation European Young Investigator Award in 2007 and the National Scientific Technological Research Council Distinguished Young Scientist Award of Turkey in 2009. 